\title{
Die Richtung der Teilungswand in Pflanzenzellen.
}

Von K. Giesenhagen.

(Mit 11 Abbildungen im Text.)

In der neuesten Auflage des Bonner Lehrbuchs der Botanik findet sich über das Problem der Anordnung der Scheidewände in sich teilenden Pflanzenzellen die folgende Bemerkung. ${ }^{1}$ ): „Während Sachs: die rechtwinkelige Schneidung zum obersten Prinzip des Verlaufs der Scheidewände erhob, suchten Errera und Berthold nachzuweisen, daß die Krümmung: und der Ansatz der Seheidewände: denselben Gesetzen folge wie bei gewichtslosen : Flüssigkeitslamellen. Die Zellmembran habe im Augenblick ihrer Entstehung das : Bestreben diejenige Gestaltung anzunehmen, wie eine gewichtslose Flüssigkeitslamelle (etwa aus Seifenwasser) sie unter denselben Bedingungen annehmen würde".

Jost sagt in seinen Vorlesungen über Pflanzenphysiologie: ${ }^{2}$ nachdem er die ältere einschlägige Literatur diskutiert hat, über den gleichen Gegenstand das Folgende:

„So verlockend also auch auf den ersten Blick die Zurückführung. der Zellteilung: auf die Gesetze des Gleichgewichtes: von Flüssigkeitslamellen ist, so können wir doch dieselbe nicht akzeptieren; wir müssen uns damit begnügen, die große Ähnlichkeit zwischen beiden Erscheinungen hervorzuheben und gestehen, daß uns die Ursachen dieser. Über: einstimmung noch ganz unklar sind."

Gegenüber diesen Äußerungen hervorragender Fachgenossen erscheint es mir nicht überflüssig darauf hinznweisen, daß ich bereits vor Jahren. in : meinen Studien über Zellteilung im Pflanzenreiche ${ }^{3}$ ) an der Hand der Beobachtungstatsachen eine mechanische Erklärung für die Richtung der Teilungswand in allen typischen Fällen gegeben habe und zugleich gezeigt habe, wie die nichttypischen Fälle, die den früheren Bearbeitern des Problems so viele Schwierigkeiten bereiteten, sich zwanglos erklären lassen aus den besonderen Umständen, unter denen sie in die Erseheinung treten Es kann nicht meine Absieht und Aufgabe sein, an dieser. Stelle den Gańg der Beweisführung zu rekapitulieren, auf die sich meine Anschaung über das Zustandekommen der gesetzmäßigen Scheidewandstellung begründet; ich. will nur in Kürze diese Anschaunng - selber darzulegen suchen, um dem Leser einige auf die-

1) Strasburger, Noll, Sehenck, Karsten, Lehrbuch d. Botanik, 9. Aufl., pagr: 134: Jena 1908 .

2) 2. Aufl., pag. 316. Jena 1908.

3) Fr. Grub's Verlag.: Stuttgart 1905. 
selbe bezügliche Experimente vorführen zu können, die zur Stütze meiner Lehre dienen und vielleicht als Vorlesungsversuche Verwendung finden können. Bezüglich aller Einzelheiten muß ich auf meine oben zitierte, im Buchhandel erschienene Arbeit verweisen.

Die älteren Autoren Hofmeister, Sachs, die sich mit dem Problem von der Richtung der Scheidewände befaßt haben, suchten zunächst nicht nach einer mechanischen Ursache für die Erscheinung, ihnen lag in erster Linie daran, aus den beobachteten Einzelfällen das Naturgesetz $\mathrm{zu}$ formulieren, dessen Walten in der erkennbaren Regelmäßigkeit der Zellwandstellung zum Ausdruck kommt. So kam Hofmeister ${ }^{1}$ zu dem Satz: „Die teilende Wand steht ausnahmslos senkrecht zur Richtung des stärksten vorausgegangenen Wachstums der Zelle“, während Sachs ${ }^{2}$ ) fand, daß die neue Teilungswand sich in der Regel rechtwinkelig an die Mutterzellwand ansetzt.

Auch Berthold und Errera gaben zunächst nur einen prägnanteren Ausdruck für das Naturgesetz, wenn sie behaupteten, daß die Stellung der Scheidewand in jedem Falle der Gleichgewichtslage einer gewichtslosen Flüssigkeitslamelle entspricht, die an der betreffenden Stelle in dem Hohlraum der Mutterzelle ausgespannt wäre. Verlockt durch die volle Übereinstimmung der Zellwandstellung mit den von dem Physiker Plateau und seiner Schule rechnungsmäßig entwickelten Gesetzen für die Gleichgewichtslage solcher Flüssigkeitshäute ging aber Errera weiter, indem er ohne Bedenken für das Zustandekommen dieser gesetzmäßigen Stellung ' der Zellwand die gleichen mechanischen Ursachen verantwortlich machte, die die Stellung der Seifenwasserlamelle bedingen. Errera war also der Meinung, die junge Zellwand sei eine Flüssigkeitsmembran, die unter den gegebenen Umständen dem Einfluß der Schwere entzogen ist und nur den in ihr gegebenen Spannungen gehorchend die ihr in dem Hohlraum der Mutterzelle zukommende Gleichgewichtslage aufsucht. Die Einwände, die Zimmermann ${ }^{3}$ ) gegen diese Auffassung geltend gemacht hat, sind unwiderlegt geblieben und auch durch die Arbeiten ${ }^{4}$ ) de Wildemans nicht im geringsten entkräftet worden. Es unterliegt keinem Zweifel, daß die Stellung der jungen Zellwand in der sich teilenden Zelle nicht bestimmt werden kann durch die in ihr auftretenden Flächenspannungen; denn bevor die

1) Hofmeister, Handb. d. physiolog. Botanik, Bd. I. Leipzig 1867.

2) Sachs, Gesammelte Abhandlungen, Bd. XXXIX u. XL. Leipzig 1893.

3) Zimmermann, Beiträge zur Morphologie und Physiologie der Pflanzenzelle, Heft 2, pag. $159 \mathrm{f}$. Tübingen 1891.

4) De Wildeman, Etudes sur l'attache des cloisons cellulaires. Brüssel 1893. 
junge Zellwand rings an die Mutterzellwand angeschlossen ist, können diese Spannungen nicht in Wirksamkeit treten, und wenn erst der Anschluß an die Mutterzellwand erfolgt ist, so ist damit eine Verschiebung $\mathrm{zu}$ einer angestrebten Gleichgewichtlage hin bereits ausgeschlossen.

Meine Anschauung über das Zustandekommen der gesetzmäßigen Stellung der Teilungswand ist nun die folgende: Der jungen Teilungswand wird ihre Lage angewiesen durch die Lagerung der beiden Tochterzellen, zwischen denen sie sich bildet. Die Lagerung dieser beiden Tochterzellen in dem Hohlraum der Mutterzelle wird bedingt durch die aus der Kohäsion resultierende Oberflächenspannung. Jede der beiden Tochterzellen hat das Bestreben, in dem von der Mutterzellwand umschlossenen Hohlraum diejenige Gestalt anzunehmen, in der sie die kleinste mögliche Oberfläche besitzt. Da die Tochterzellen den Hohlraum der Mutterzelle ganz ausfüllen und deshalb in ihrer Gestalt von der Form dieses Hohlraums abhängig sind, so kann sich das aus der Kohäsion abzuleitende Bestreben zur Verkleinerung der Oberfläche nur auf den nicht mit der Mutterzellwand in Berührung stehenden Teil ihrer Oberfläche, d. h. auf die freie Berührungsflăche der beiden Tochterzellen beziehen. In dieser Berührungsfläche wirkt demnach bei einer Halbierungsteilung diè Oberflächenspannung beider Tochterzelleiber glếichsinnig dahin, daß sie eine Fläche minimae areae wird, oder ganz allgemein ausgedrückt, daß die potentielle Energie des Systems ein Minimum wird. Die dieser Forderung entsprechende Gleichgewichtslage wird von den beiden Tochterzellen eingenommen, bevor die Teilungswand ausgebildet ist und die an der Berührungsfläche sich bildende Teilungswand befindet sich also, wenn sie sich an die Zellwand der Mutterzelle ansetzt, bereits in der Lage, die nach Plateau's Berechnungen der Gleichgewichtslage einer gewichtslosen Flüssigkeitslamelle entspricht.

Wenn meine Anschauung richtig ist, wenn die Einstellung der Berührungsfläche der Tochterzellen ein rein mechanischer Vorgang ist, bei dem der Zelleib als lebende Substanz nicht anders mitwirkt wie ein Flüssigkeitstropfen von gleicher Dichte, so muß sich auch der Vorgang an leblosem Material nachahmen lassen. Es handelt sich nur darum, die gleichen physikalischen Bedingungen $\mathrm{zu}$ schaffen, die bei der Zellteilung den Vorgang beherrschen. Es handelt sich also im einfachsten Falle einer Halbierungsteilung darum, in einen Hohlraum von der Gestalt einer Pflanzenzelle zwei gleiche in sich kohärente, aber sich miteinander nicht mischende Körper einzuschließen, die etwa die Bildsamkeit eines zähflüssigen Plasmatropfens besitzen, und die mit 
ihrem Kubikinhalt den gegebenen Hohlraum vollständig ausfüllen. In einem solchen System - das ist nun die Forderung meiner Theorie muß die Berührungsfläche der eingeschlossenen Körper der Lage entsprechen, die unter ähnlichen räumlichen Verhältnissen die Teilungswand einer Mutterzelle einnehmen würde. Gelingt es im Experiment zu zeigen, daß die in einen Hohlraum eingeschlossenen Körper eine Lagerung einnehmen, in der ihre Berührungstläche der Stellung entspricht, die unter gleichen Formverhältnissen die Teilungswand der Zellen einnehmen würde, so ist bewiesen, daß die Teilungswand selber entgegen der Ansicht Errera's bei dem Zustandekommen der gesetzmäßigen Richtung nicht mitzuwirken braucht, daß vielmehr die Teilungswand durch die Zellkörper passiv in ihre gesetzmäßige Richtung gebracht wird.

Um den experimentellen Nachweis für die Richtigkeit meiner mechanischen Erklärung der Stellung der Scheidewand in den sich teilenden Pflanzenzellen zu erbringen, habe ich mit luftgefüllten Gummiblasen operiert. Die Gummihüllen der bekannten Kinderluftballons, wie sie überall auf Messen und Märkten feilgeboten werden, liefern dafür ein leicht erhältliches, wenn auch nicht für alle Fälle ausreichendes Material. Ein Schüler von mir, Herr Diplomingenieur Habermehl, hat nach meiner Angabe mit solchen Gummiballons einige Versuche angestellt, und wird darüber in einer demnächst erscheinenden Arbeit, in der auch die theoretischen Grundlagen der Versuchsanstellung eingehender erörtert werden sollen, berichten. Hier soll nur mitgeteilt werden, wie die Experimente anzustellen sind, und wiefern ihr Ergebnis als eine Stütze meiner Erklärung für das Zustandekommen der regelmäßigen Seheidewandstellung in Pflanzenzellen angesehen werden kann.

Man blase zwei derartige Gummiballonhüllen ungefähr bis zur Walnußgröße auf, binde sie fest $\mathrm{zu}$ und bringe sie in eine genügend weithalsige kugelige Glasflasche aus nicht zu dünnem Glas, deren Durchmesser ca. $10-15 \mathrm{~cm}$ betragen mag. Der Flaschenhals wird darauf mit einem einmal durchbohrten Gummistopfen verschlossen, durch dessen Bohrung eine Glasröhre mit Zweiweghahn geführt ist. Wir haben nun in dem einer kugelförmigen Mutterzelle entsprechenden Hohlraum der Flasche zwei sich nicht mischende Körper, die infolge der Oberflächenspannung die Kugelform zu erhalten streben wie ein von der Schwerkraft unabhängiger Flüssigkeitstropfen und die ähnlich wie ein Flüssigkeitstropfen durch äußere Kräfte leicht deformiert werden können. Daß die im Flüssigkeitstropfen aus dem Kohäsionsdruck resultierende Oberflächenspannung hier durch die Elastizität der aufgeblasenen Gummihülle ersetzt ist, ändert an den Verhältnissen nichts Wesentliches, wenn nur 
die Elastizität in der ganzen Gummihaut gleichmäßig ist. Ich will gleich hier bemerken, daß das bei den. Gummihüllen der Kinderluftballons nicht immer genügend der Fall ist. Man erkennt die Ungleichmäßigkeit, wenn man die Ballons vorher versuehsweise stark aufbläst. Wenn ein Teil der Wand dabei weniger gedehnt wird, so muß einmal die ungleiche Spannung in der Oberfläche und ferner auch der größere Widerstand, den die dichteren Stellen der Gummihaut der Deformierung entgegenstellen, bei den Experimenten zu abweichenden Ergebnissen führen. Wir verwenden deshalb zunächst nur Gummihüllen, die beim Aufblasen möglichst gleichmäßig: kugelig anschwellen. Wenn wir nun in dem System noch die Bedingung erfüllen, daß die zwei eingeschlossenen Körper mit ihrem Kubikinhalt den gegebenen Hohlraum ganz ausfüllen, so sind diejenigen physikalischen Verhältnisse geschaffen, aus denen in der sich teilenden

\begin{abstract}
Versuchsanordnung. Die kräftigwirkende Wasserstrahl-Luftpumpe $L$, die bei $W$ an die Wasserleitung angeschlossen ist, steht durch den Druckschlauch $D$ mit dem Rezipienten $R$ in Verbindung. Letzterer ist im vorliegenden Falle kugelförmig, trägt im durchbohrten Gummistopfen eine Glasröhre mit Glashahn $H$ und wird am Halse von einem Klammerstativ gehalten. $X X$ bezeichnen die Enden des Saugrohrs der Pumpe und des Druckschlauches, die durch ein außerhalb der Figur liegendes Schlauchstück verbunden sind.
\end{abstract}

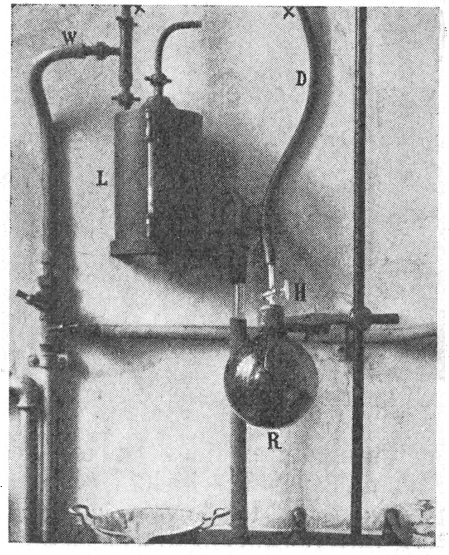

Fig. 1.

Zelle die Stellung der Teilungswand mechanisch erklärt werden muß. Wir erfüllen, ohne die übrigen Verhältnisse in unserem System zu ändern, diese letzte Bedingung in ausreichendem Maße dadurch, daß wir das Glasrohr in dem Stopfen bei geöffnetem Hahn durch einen Druckschlauch mit einer Luftpumpe verbinden (s. Fig. 1) und solange evakuieren, bis die beiden Gummiballons ringsum mit der Glaswand der Flasche in Berührung getreten sind. Durch Schließen des Glashahns wird der dabei : erreichte Zustand im Innern des Systems fixiert. Dünne Glaskolben ertragen den durch das Evakuieren entstehenden Oberflächendruck nicht; ich muß vor deren Verwendung warnen. Mir zersprang ein Kolben mit dumpfem Knall in kleine Splitter, die mit Gewalt umhergeschleudert wurden. Ich: kam durch glücklichen Zufall mit unbedeutenden Vèrletzungen an der Hand davon, im unglücklichen Falle 
könnten aber Gesicht und Augen in ernste Gefahr kommen. Die Berührungsfläche der beiden Ballons in der evakuierten Glaskugel, die durch die gedehnten Gummihäute deutlich hindurchscheint, erweist sich als eine Ebene, die den kugelförmigen Hohlraum annähernd halbiert.

Die Fig. 2 zeigt einen kugelförmigen Rezipienten, der zwei gleichgroße Gummiblasen (eine blaue und eine rote) enthält, vor $(A)$ und nach $(B)$ dem Evakuieren. Die Lage der Bälle in $A$ wird natürlich durch die Schwerkraft bestimmt; wenn man die Glaskugel neigt, so gleiten sie an der gut mit Paraffin geschmierten Wand zum tiefsten Punkt der Kugel abwärts. Wenn man evakuiert, so schwellen die Bälle an und verlassen dabei ihren Platz, indem sie immer in Berührung miteinan-

Fig. 2.

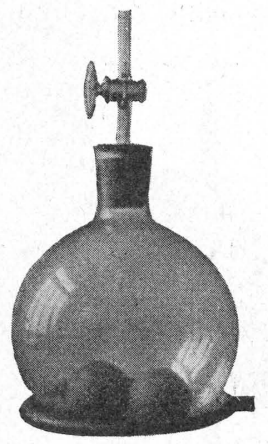

$A$

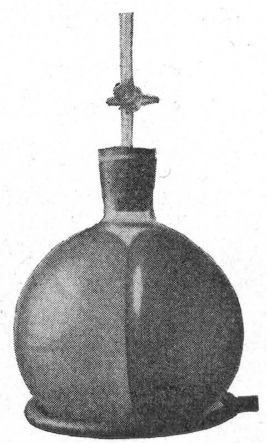

$B$
Der kugelförmige Rezipient ist mit '2 gleichgroßen Gummiblasen beschickt $(A)$. Beim Evakuieren werden beide Kugeln zu Halbkugeln deformiert, ihre Berührungsfläche ist eine Ebene, die den Hohlraum der Kugel halbiert.
Fig. 3.

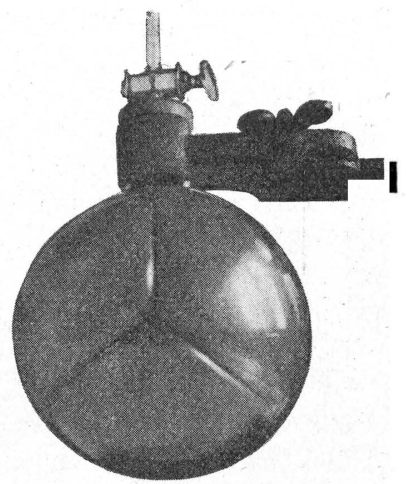

Kugelförmiger Rezipient mit 4 Gummibällen, die beim Evakuieren die tetraedrische Lagerung angenommen haben und sich in ebenen Flächen berühren, die im Mittelpunkt der Kugel zusammentreffen.

der an der Wand der Glaskugel aufwärts gleiten, bis sie im horizontalen Durchmesser der Kugel liegen. Von diesem Moment an ist die Wirkung der Schwerkraft auf die Lage der Bälle ausgeschlossen, sie befinden sich bei jeder beliebigen Stellung der Glaskugel in einer Ruhelage. Bei weiterem Evakuieren platten sich die Bälle durch den Druck an ihrer Berührungsfläche $a b$, indem sie sich gleichzeitig der Wand der Glaskugel mehr und mehr anschmiegen. Das in $B$ dargestellte Endstadium zeigt die Wand der beiden Ballons nach außen rings der Glaskugelinnenwand angepreßt. Die Berührungsfläche aber ist eine Ebene, und zwar eine Halbierungsebene der Kugel, die ja in diesem einfachsten Falle der Gleichgewichtslage einer gewichtslosen Flüssigkeitslamelle entspricht. Eine in dieser Berührungsfläche ausgeschiedene Wand würde 
also die Gleichgewichtslage einer gewichtslosen Flüssigkeitslamelle bekommen, aber ohne daß dabei irgendwelche in ihr selbst wirkende Spannungskräfte mitzuwirken brauchen, ohne daß wir sie uns als gewichtslose Flüssigkeitslamelle vorzustellen brauchen, die sich ihre Gleichgewichtslage selbst aufsucht. Die Lage und Gestalt, welche die Halbierungswand kugeliger Pflanzenzellen überall aufweist, ist demnach nicht bedingt durch die Gleichgewichtslage, welche sie als gewichtslose Flüssigkeitslamelle einnehmen müßte, sondern durch die Gleichgewichtslage, die die Plasmaleiber der beiden Tochterzellen infolge ihres Kohäsionsdruckes resp. der daraus resultierenden Oberflächenspannung bereits vor dem Auftreten der Teilungswand einnehmen.

Bringt man statt der zwei zugleich vier zu Walnußgröße aufgeblasene Gummiballons in die Flasche, so ordnen sich dieselben bei dem

Fig. 4.

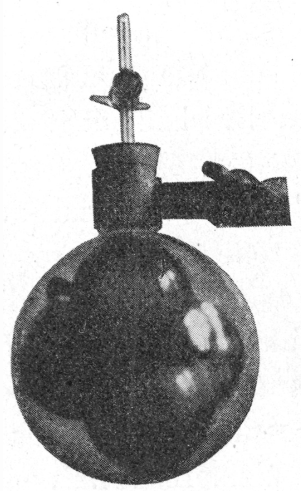

A

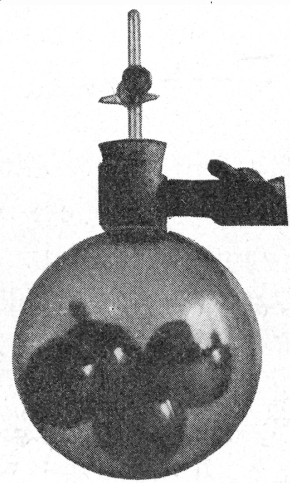

$B$
Der mit 4 gleichen Gummibällen beschickte kugelförmige Rezipient $A$ vor dem Evakuieren, $B$ nachdem die Bälle durch ihre Größenzunahme eben in die tetraedrische Lage gedrängt sind.
Fig. 5,

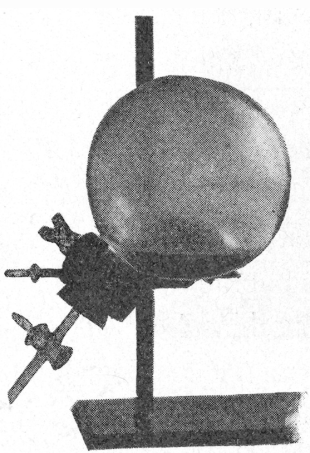

Kugelförmiger Rezipient mit 2 ungleichgroßen Gummiblasen beschickt, die nach dem Evakuieren eine uhrglasförmige Berührungsfläche aufweisen.

Evakuieren, wie Fig. 3 zeigt, in der bekannten tetraedrischen Lagerung an, die bei simultaner Vierteilung in Pollen- und Sporenmutterzellen und Tetrasporangien so häufig $\mathrm{zu}$ finden ist. Die Berührungsflächen der Ballons sind Ebenen, die sich annähernd im Mittelpunkt des Hohlraums schneiden und mit einander annähernd gleiche Winkel bilden.

Dieses Endstadium zeigen auch die Gummiblasen in dem in Fig. 1 mit abgebildeten kugelförmigen Rezipienten.

Man kann bei diesem Versuch ganz besonders deutlich verfolgen, wie die Erreichung der endlichen Ruhelage abhängig ist von der gegenseitigen Beeinflussung der Gummiblasen. Fig. 4 zeigt aufeinanderfolgende Phasen des gleichen Versuches. Bei $A$ liegen die Bälle vor 
dem Evakuieren des Rezipienten in zufälliger Anordnung nicht in der Gestalt eines Kugelhaufens (nicht tetraedrisch) sondern nahezu in einer Ebene. Beim Evakuieren gleiten die Bälle infolge ihrer Größenzunahme aneinander und an der gut mit flüssigem Paraffin geölten Glaswand hin und erreichen, sobald ihre Größe entsprechend zugenommen hat, die Anordnung eines Kugelhaufens, wobei alle vier Bälle die Glaswand berühren, so daß nunmehr auch hier die Ballgruppe in ihrer Lagerung von der Wirkung der Schwere unabhängig geworden ist. Diese Phase ist durch Schließen des Glashahns fixiert und in Fig. $4 B$ photographisch aufgenommen worden.

Wenn man nicht die Glaswand und die Bälle selbst durch flüssiges Paraffin gut gleitend macht, so werden die Bälle beim Aufschwellen länger in ihrer zufälligen gegenseitigen Lage festgehalten und es kommen schließlich ganz unregelmäßige Lagerungen zustande. Die Bedingung, daß die Teile der in der Berührungsfläche aneinandergrenzenden Oberflächenstücke der Tochterzellen leicht gegeneinander verschiebbar sind, darf aber wohl bei der flüssigen Beschaffenheit des Protoplasmas in allen normalen Fällen für erfüllt gelten.

Noch ein Versuch kann leicht mit dem gleichen Material angestellt werden. Wir bringen einen zu Walnußgröße aufgeblasenen und einen etwa nur ein Viertel soweit aufgeblasenen Ballon in die Kugelflasche und sehen, wie Fig. 5 zeigt, beim Evakuieren die Berührungsfläche der beiden ungleichen Ballons das von der Keimung der Sporen von Equisetum, Selaginella usw. bekannte Bild der „uhrglasförmigen Teilungswand" darbieten. Dabei geht der kleinere Ball, wenn er vielleicht anfangs infolge des geringen Aufblasens nicht ganz kugelig war, zunächst in die Kugelform über, bis der Durchmesser der beiden Gummiblasen zusammen gleich dem Durchmesser des Rezipienten ist. Dann beginnt bei weiterem Evakuieren die Abplattung der kleineren Kugel. Im Endstadium bildet die Berührungsfläche entsprechend den Plateau'schen Regeln eine Fläche von konstanter Krümmung, die rechtwinkelig an die Glaswand ansetzt.

Man muß sich bei den eben geschilderten Versuchen immer so einrichten, daß̉ eine Berührungsfläche der Ballons in der Nähe des inneren Endes des Glasrohres zu liegen kommt. Man kann sonst die Evakuierung nicht weit genug fortführen, da der abgesaugten Luft durch den sich anpressenden Gummibállon der Ausweg versperrt wird. Das gleiche Hindernis macht sich bei der Versuchsanstellung bemerkbar, wenn man die Ballons in einem zylindrischen Hohlraum unterbringt, um die Anordnung der Berührungsflächen senkrecht zur Längsachse 
des Zylinders zu demonstrieren. Sobald bei der Evakuierung die beiden Ballons bis zur Berührung der Glaswand des Zylinders angeschwollen sind, ist das zwischen ihnen eingeschlossene Luftquantum abgesperrt und durch Absaugen nicht mehr zu entfernen. Man könnte wohl für diesen Versuch prismatische Gläser verwenden, deren Längskanten ziemlich lange noch ein Abströmen der zwischen den Ballons eingeschlossenen Luft gestatten werden. Aber solche prismatischen Gläser werden nicht überall in geeigneter Größe zur Verfügung stehen und deshalb mag ein anderer Behelf hier empfohlen sein. Man legt in das Zylinderglas, das als Rezipient dienen soll, einen runden Glasstab von der Länge des Zylinders. Durch den Gummistopfen wird von der kleineren Fläche des Stopfens her ein nahezu rechtwinkelig gebogenes Glasrohr geführt, an das oben ein Glashahn mit Gummischlauch angesetzt wird. Beim Einsetzen des Gummistopfens in den Hals des Rezipienten ist darauf $\mathrm{zu}$ achten, daß das innere Ende des gebogenen Glasrohrs an der Wand des Zylinders in der Nähe des Glasstabes zu liegen kommt, damit möglichst lange die in den Winkeln längs des Glasstabes fortrückende Luft nach außen abgesaugt wird ${ }^{1}$ ). Beschickt man den 'so vorbereiteten zylindri-

Zylindrischer Rezipient, in dem die Begrenzungsflächen der Gummiballons beim Evakuieren sich senkrecht zur Längsachse des Zylinders anordnen.

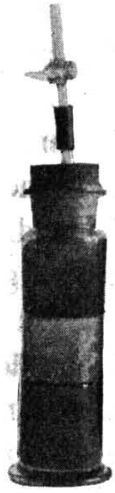

Fig. 6. schen Rezipienten mit walnußgroßen Bällchen, so nehmen dieselben beim Evakuieren Trommelform an derart, daß ihre Berührungsflächen, wie Fig. 6 erkennen läßt, eben und zur Längsachse des Zylinders senkrecht gerichtet sind.

Auch bei diesem Versuch ist es erforderlich, daß Gummibälle mit gleichmäßiger Wand ausgewählt werden und daß Glas und Bälle gut gefettet sind.

Man kann in dem zylindrischen Rezipienten auch leicht Längsteilungen erzielen, wenn man zwischen den zu Walnußgröße aufgeblasenen Ballons zwei weniger stark aufgeblasene Bälle einschaltet, die nebeneinander Platz haben. Noch besser läßt sich das erweisen, wenn man längliche Gummiblasen statt der kugeligen verwendet, d. h. also Schläuche, deren Wandung der Dehnung in der Längs- und Querrich-

1) Die Verwendung des Glasstabes wurde von Herrn Dipl.-Ing. Habermehl ersonnen und bei seinen Versuchen brauchbar befunden. Ich habe die Methode durch die gleichzeitige Anwendung des gebogenen Glasrohrs für eine Reihe von weiteren Versuchen adaptiert. 
tung ungleichen Widerstand entgegensetzt, oder was dasselbe sagt, Körper, an denen die Oberflächenspannung ungleiche -Werte aufweist. Es wird sich zunächst fragen, ob damit nicht Verhältnisse eingeführt werden, die in der Natur bei den Plasmaleibern der Pflanzenzellen kein Analogon haben. Wenn das Protoplasma immer in den Zellen die Natur eines Flüssigkeitstropfens aufwiese, von durchweg gleicher Dichte, so müßte sich das Protoplasma kugelig abrunden sobald der formbestimmende Einfluß der festen Zellwand aufgehoben wird. Bei vorsichtiger Plasmolyse müßte sich also das Plasma zu einem annähernd kugeligen und durch das eigene Gewicht etwas abgeplatteten Tropfen zusammenziehen. Das ist nun in der Tat der Fall in allen daraufhin untersuchten Zellen und diese Tatsache gibt uns gerade das Recht, das Plasma als flüssige Substanz anzusehen, und zur Erklärung seines Verhaltens bei der Zellteilung die Gesetze heranzuziehen, die die physikalischen Vorgänge in Flüssigkeiten beherrschen. Aber andererseits kann die Annahme nicht von der Hand gewiesen werden, daß die Lebensvorgänge in dem Protoplasma speziell in der Hautschicht des Protoplasten zeitweilig lokale Veränderungen der Dichtigkeit und damit der Oberflächenspannung hervorrufen und vorübergehend erhalten können, die dann selbstverständlich einen Einfluß auf die Gestalt des von der Zellwand zurückweichenden Protoplasten ausüben müssen. Pfeffer ${ }^{1}$ ) gibt die Möglichkeit solcher physiologischen Beeinflussung der Oberflächenspannung zu. Er schreibt: ,an und in dem Protoplasten herrschen die rein physikalischen Gesetze, jedoch ist zu bedenken, daß im Vergleich $\mathrm{zu}$ einer vollkommen homogenen Flüssigkeit weitgehende Abweichungen möglich sind, weil der Protoplast durch die physiologische Tätigkeit Potentialdifferenzen (der Oberflächenspannung usw.) schaffen und erhalten, und ferner die Kohäsion seiner Teile selbsttätig erhöhen kann".

Ich glaube Andeutungen für das Vorhandensein derart entstandener Anomogenität in der Verteilung der Oberflächenspannung in den lebenden Zellen darin sehen zu dürfen, daß die Protoplasten bei der Plasmolyse durchaus nicht immer auf direktem Wege der von den physikalischen Gesetzen geforderten Kugelform zustreben, sondern zunächst in ihrer Form noch die Gestalt, d. i. insbesondere das Verhältnis der Länge und Breite ihrer Zellkammer, erkennen lassen. Die in Pfeffers Handbuch I, pag. 116 abgebildete Fig. $10 b$ zeigt den plasmalysierten Protoplasten in Eiform, wobei die längere Achse der längsten Dimension

1) Pfeffer, Pflanzenphysiologie, Il, pag. 47. 
der Zellkammer gleichgerichtet ist. Ich sah an den in starker Streckung begriffenen Berindungszellen jugendlicher Sprosse von Chara ceratophylla, die nach kurzer Einwirkung einer plasmolytisch wirkenden Lösung fixiert und dann zu Mikrotomschnitten verarbeitet waren, die in Fig. 7 dargestellten Bilder. Die zusammengezogenen Protoplasten ließen in ihrer Umrißform noch die ehemalige, durch die Zellwạnd ihnen aufgeprägte Gestaltung erkennen.

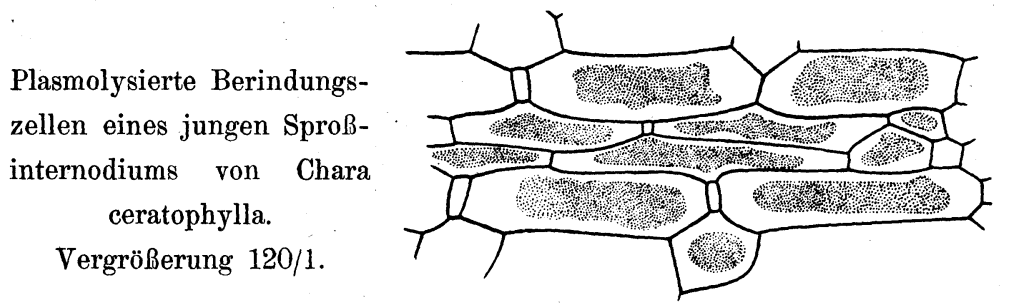

Fig. 7.

Es ist wohl möglich, daß auch in diesen Zellen die plasmolysierten Protoplasten nach einiger Zeit die Kugelform angenommen haben würden oder doch wenigstens sich ihr genähert haben würden, soweit es der Raum gestattet. In jungen lebenden Blattzellen wird dieses Stadium, wie ich wenigstens in einigen Fällen konstatieren konnte, schließlich erreicht, offenbar indem die vorhàndenen Spannungsdifferenzen in dem Plasmatropfen allmählich ausgeglichen werden.

Auch die bekannte Tatsache, daß in schlauchförmigen Zellen das Plasma bei der Plasmolyse bisweilen in einzelne Tropfen zertrennt wird, spricht wohl dafür, daß die Oberflächenspannung nicht homogen war. Besonders scheint mir auch das ausgesprochene Spitzenwachstum zahlreicher Zellfäden bei Pilzen, Pollenschläuchen, Haarzellen und Rhizoiden dafür zu sprechen, daß die der Ausdehnung entgegenwirkende Oberflächenspannung an der fortwachsenden Spitze geringer ist als an den Flanken des langgestreckten Protoplasten. Ganz besonders naheliegend erscheint es mir anzunehmen, $d a ß$ an der eben neu entstandenen Trennungsfläche zweier Tochterzellen andere Dichtigkeits- und Spannungsverhältnisse gegeben sind als in den der alten Zellwand anliegenden Teilen, des Zellumfanges, in denen wir ja überall eine Hyaloplasmaschicht mit bestimmten Struktureigentümlichkeiten und mit besonderen physiologischen Funktionen anzunehmen haben. Ich glaube aus derartigen Unterschieden in der Oberflächenspannung des Protoplasten, und zugleich aus einer die Verschiebung und Deformierung erschwerenden Adhäsionswirkung zwischen der alten Zellwand und dem Protoplasma, gewisse mir vereinzelt vorgekommene Fälle erklären zu können, in 
denen scheinbar bei der Zellteilung von den Tochterplasmen statt der nächstliegenden eine andere Gleichgewichtslage aufgesucht wurde. Doch das gehört nicht zum Gegenstande dieser Mitteilungen. Hier sollte nur nachgewiesen werden, daß die Annahme von anomogener Oberflächenspannung in gewissen Protoplasten durch Beobachtungstatsachen gestüzt wird. Das oben erwähnte Verhalten des Protoplasmas langgestreckter Zellen bei der Plasmolyse gibt uns das Recht, auch schlauch- oder sackförmige Gummiblasen zu unseren Versuchen zu benutzen, um eben die physikalischen Verhältnisse nachzuahmen, die in solchen gestreckten Mutterzellen bei der Zellenteilung vorhanden sind. Gummihäute, die beim Aufblasen zunächst Wurst- oder Schlauchform annehmen, werden gleichfalls als Kinderspielzeug fabriziert, nur ist der dazu verwendete Gummi meist von minderer Qualität. Ich habe deshalb mit Vorteil ein anderes Material verwendet, das aus den feinsten Gummisorten hergestellt wird, nämlich die überall käuflichen Gummipräservativs.

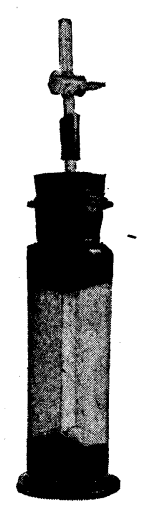

Fig. 8.
Zylinder mit

2 kugeligen (roten)

Gummiblasen oben und unten, und 2 länglichen (weißen) Gummiblasen in der Mitte. Die letzteren haben beim

- Evakuieren eine längsgerichtete ebene Berührungsfläche gebildet.

In den für den vorhergehenden Versuch verwendeten Glaszylinder mit eingelegtem Glasstab brachte ich, um den Einfluß der Unregelmäßigkeit des Glasbodens auszuschalten, zunächst einen runden Gummiballon, darauf wurden zwei gleichlange Schläuche und endlich obenauf, zur Abgleichung der Unregelmäßigkeiten am Stopfen, wieder ein runder Gummiballon eingebracht. Beim Evakuieren erfüllten die beiden kugeligen Gummibälle oben und unten trommeförmige Abschnitte des Zylinders. Den größeren mittleren Teil aber füllten die beiden länglichen Gummiblasen nebeneinander in der Weise aus, daß eine ebene, die Längsachse des Zylinders in sich aufnehmende Längswand gebildet wurde, wie aus der Fig. 8 ersichtlich ist.

Ich will nun zum Schluß noch auf einige abnorme Zellteilungsfälle eingehen, die sich den von Hofmeister, Sachs, Errera angegebenen Regeln nicht unterordnen, und deshalb diesen Autoren und denen, die sich auf ihren Standpunkt stellten; bei ihren Erklärungsversuchen unüberwindliche Schwierigkeiten darboten, nämlich die schiefstehenden Wände der Moosrhizoiden und die sohlenförmigen Wände in den Rhizoiden der Characeen. Bei den Moosrhizoiden treten, wie bekannt, schiefstehende Wände in großer Żahl auf. Bisweilen bilden sie doppelt gebogene Flächen, aber auch nahezu ebene Flächen, die an zwei gegenüber liegenden Punkten spitzwinklig an die Zellenwand der 
zylindrischen Mutterzelle anschließen, kommen sicher vor. Man findet alles nähere darüber in Goebel's Organographie ${ }^{1}$ ) übersichtlich zusammengestellt und kritisch beleuchtet. De Wildeman ${ }^{2}$ ), der Errera's Anschauungen von der Mitwirkung der jungen Teilungswand auch in diesen Fällen zu stützen sucht, gibt an, daß die schiefen Wände der Moosrhizoiden immer als doppelt gebogene Fläche angelegt werden, und daß die extremen Fälle, in denen ein schiefwinkliger Ansatz an die Mutterzellwand beobachtet wird, durch nachträgliche Wachstumsverschiebungen zustande kommen.

Ich habe in meiner Arbeit über die Zellteilung ${ }^{3}$ ) einige direkte Beobachtungen über den Teilungsproze $\beta$ bei Moosrhizoiden mitgeteilt. Von den scheinbar abweichenden Beobachtungsresultaten an anderen Moosen, über die $\mathrm{Némec}^{4}$ ) nebenbei in einer Anmerkung berichtet, habe ich Notiz genommen, ohne darin eine Widerlegung meiner Angaben finden zu können. Nach meinen Beobachtungen ist gerade entgegen der Annahme de Wildeman's, die ebene Trennungsfläche das Primäre; die doppelt gebogenen Flächen erweisen sich als Übergangsflächen zwischen der schiefgestellten und der senkrecht zur Längsachse gestellten Teilungsebene. Um das Zustandekommen solcher doppelt gebogenen Übergangsflächen experimentell $\mathrm{zu}$ erweisen, habe ich in meinen Studien über die Zellteilung den folgenden Versuch angegeben ${ }^{5}$ ). Man füllt in ein Reagenzglas eine schwach sirupdicke Lösung von Gummi arabicum einige Zentimeter hoch ein und gießt vorsichtig über dieselbe eine ebenso hohe Schicht von Kanadabalsam in Toluol gelöst, welcher annähernd die gleiche Konsistenz besitzt- wie die Gummilösung. Neigt man, nachdem die Berührungsfläche der beiden sich nicht mischenden Flüssigkeiten in dem aufrecht stehenden Reagenzglas zur Ruhe gekommen ist, das letzte plötzlich um etwa $45^{\circ}$, so geht die ebene Berührungsfläche der Flüssigkeiten in eine zweifach gekrümmte Fläche über, welche in der Form ganz der bei Moosrhizoiden gewöhnlichen Querwandfläche entspricht. Erst allmählich, und zwar je nach der Konsistenz der Lösungen und dem Grade ihrer Adhäsion an die Glaswand in verschieden langen Zeiträumen wird diese Übergangsfläche in die horizontale Ebene übergeführt, welche in der neuen Lage des Reagenzglases schief zu der Längsachse desselben steht. In der schematischen Fig. 9 ist die Ruhelage der Berührungsfläche in $a$ und $e$

1) Goebel, Organographie, pag. $340 \mathrm{f}$.

2) De Wildeman, Études sur l'attache des cloisons cellulaires. Brüssel 1893.

3) A. a.' 0., pag. 71.

4) Némec, 1906.

5) A. a. 0., pag. 73 . 
bei vertikaler, in $c$ bei schräger Stellung des Glases dargestellt; $b$ ist eine Übergangsfläche, die nach der Schrägstellung des Glases zwischen beiden Ruhelagen durchlaufen wird. Die physikalischen Bedingungen für das Zustandekommen dieser Übergangslage sind klar.

Die Schwerkraft erteilt in dem geneigten Glase den Flüssigkeitsteilchen den gleichen Impuls zur Lagenänderung zur Aufsuchung der neuen Gleichgewichtslage. Der dazu nötigen Bewegung widerstrebt im Innern der Flüssigkeit allein die Kohäsin der Teilchen, in den Randpartien summiert sich damit die Adhäsion der Flüssigkeitsteilchen an der Glaswand. Es muß folglich der Widerstand im Innern der Flüssigkeit leichter, d. i. schneller überwunden werden: die mittlere Partie der Berührungsfläche eilt also in der Bewegung gegen die neue Ruhelage voraus. Dasselbe findet statt, wenn die Berührungsfläche bei Wiederaufriehtung des Glases aus der neuen Ruhelage $c$ in die erste Stellung

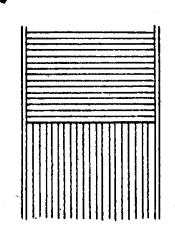

$a$

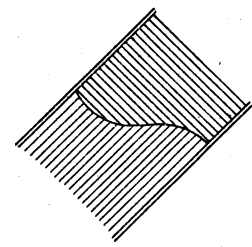

$b$

Fig. 9.
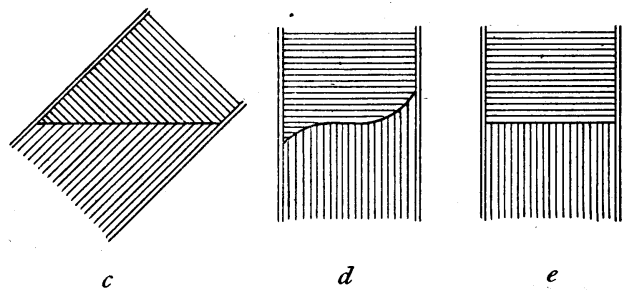
übergeführt wird; wir sehen dementsprechend nunmehr eine Fläche von der in $d$ schematisch dargestellten Form auftreten. Denken wir uns die physikalischen Bedingungen verändert, nẹmen wir an, daß die Verschiebbarkeit der Flüssigkeitsteile im Innern der Flüssigkeit größerem Widerstande begegnet als in der Peripherie, so müssen auch die peripherischen Teile der Berührungsfläche in der Bewegung vorauseilen. Wir müssen dann also bei dem Übergang aus der Ruhelage $a$ nach $c$ Übergangsflächen von der Form $d$ auftreten sehen und ebenso bei der Umwandlung der zur Achse des Glases geneigten Fläche in die dazu senkrechte Fläche Übergangsflächen von der Form $b$. Das letztere entspricht genau dem Verhalten der Querwände in den Moosrhizoiden. Ich habe in meiner Arbeit bei der Diskussion der eben geschilderten Experimente die Vermutung ausgesprochen, daß das Plasma der Moosrhizoiden in der Mitte der Berührungsfläche bei der Zellteilung dichter sei, weil dort das Baumaterial der Tochterkerne und die Spindelfasern der Kernfigur die Konsistenz beeinflussen. Die'größere Dichtigkeit würde aber in einer anomogenen Oberflächenspannung in der Berührungsfläche zum Ausdruck kommen, die in jeder augenblicklichen Lage der 
Übergangsfläche deren Gestalt bestimmt. Wenn diese Annahme richtig ist, so $\mathrm{muB}$ es auch bei geeigneter Versuchsanstellung gelingen, mit Hilfe der schlauchförmigen Gummiblasen mit anomogener Elastizität Berührungsflächen zu erzielen, die den schiefen oder doppelt gebogenen Wänden des Moosrhizoiden entsprechen. Natürlich werden die so entstehenden Berührungsflächen, da ja das Material nicht wie der lebende Plasmakörper seine innere Beschaffenheit zu ändern vermag, als einer Gleichgewichtslage der beiden Gummiblasen entsprechend dauernd bestehen bleiben, was bei den Teilungsflächen der Moosrhizoiden erst der Fall ist, wenn eine an die Mutterzellwand an schließende Teilungswand gebildet worden ist.

Es bereitet keine besonderen Schwierigkeiten, diese Forderung im Experiment zu erfüllen. Wenn man zwei schlauchförmige Gummiblasen von entsprechender Länge so in den zylindrischen Rezipienten legt, daß ihre Enden in der Mitte des Zylinders ein wenig übereinander greifen, so bekommt man das Bild einer schief zur Längsachse stehenden Teilungsfläche, wie es in Fig. 10 photographisch festgehalten ist. Hier ist nicht von rechtwinkliger Schneidung die Rede. Läßt man etwas längere Gummiblasen etwas weiter übereinander greifen, so bekommt man, wie Fig. 11 wohl ohne nähere Erläuterung erkennen läßt, die Stellung der Berührungsflächen, die der so oft abgebildeten und als Ausnahme angeführten sohlenförmigen Teilungswand in der Endzelle der Characeenrhizoiden entspricht. Indem man die Längenverhältnisse der Schläuche variiert, kann man

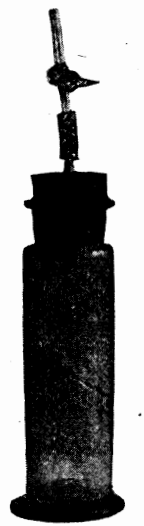

Fig. 10.

Zylindrischer Rezipient mit 2 schlauchförmigen

Gummiblasen, Gur die infolge der die infolge der anomogenen ungleichOberflächen- mäßigen spannung eine Oberflächenschief zur spannung eine Längsachse sohlenförmige stehende Be- Berührungsrührungs- fläche gebilfläche gebil- det haben. det haben. alle möglichen Übergangsformen nach Lage und Krümmung zwischen diesen beiden Flächen erzielen.

So läßt sich also zeigen, daß die oft diskutierten abweichenden Stellungen der Teilungswände in Moos- und Characeenrhizoiden, ebenso wie alle anderen in Pflanzenzellen beobachteten Stellungen der jungen Teilungswand der aus der Oberflächenspannung resultierenden Gleichgewichtslage in sich kohärenter leicht deformierbarer Körper entsprechen, die mit der Summe ihrer Volumina das Volumen eines starrwandigen Hohlraums ganz ausfüllen. 